\title{
Impact of rotation on the geometrical configurations of fossil magnetic fields
}

\author{
C. Emeriau and S. Mathis \\ Laboratoire AIM Paris-Saclay, CEA/DSM - CNRS - Université Paris Diderot, IRFU/SAp \\ Centre de Saclay, F-91191 Gif-sur-Yvette Cedex, France \\ email: constance.emeriau@cea.fr, stephane.mathis@cea.fr
}

\begin{abstract}
The MiMeS project demonstrated that a small fraction of massive stars (around $7 \%$ ) presents large-scale, stable, generally dipolar magnetic fields at their surface. These fields that do not present any evident correlations with stellar mass or rotation are supposed to be fossil remnants of the initial phases of stellar evolution. They result from the relaxation to MHD equilibrium states, during the formation of stable radiation zones, of initial fields resulting from a previous convective phase. In this work, we present new theoretical results, where we generalize previous studies by taking rotation into account. The properties of relaxed fossil fields are compared to those obtained when rotation is ignored. Consequences for magnetic fields in the radiative envelope of rotating early-type stars and their stability are finally discussed.
\end{abstract}

Keywords. magnetic fields, MHD, stars: magnetic fields, stars: rotation, stars: interiors

\section{Fossil magnetic fields in early-type stars}

Stellar magnetism is one of the key mechanisms that must be studied to understand the evolution of stars. Indeed, magnetic fields impact the structure of massive stars, their activity and winds, and the transport of angular momentum and mixing in their interiors (e.g. Mestel 1999, Maeder, Eggenberger, and Gellert in this volume). In this context, high-resolution spectropolarimetry allows us to study the large variety of stellar magnetic fields as a function of stellar parameters and evolutionary stages (e.g. Donati \& Landstreet 2009, Landstreet and Wade in this volume). In upper main-sequence stars with external radiative envelope, no correlations are found between the properties of supposed fossil fields, which are often oblique dipoles, and stellar parameters (Wade et al. 2011).

In this context, studies led by Tayler (1973) and Markey \& Tayler (1973) demonstrated that purely poloidal and toroidal fields are unstable in stably stratified zones. It was then proposed by Tayler (1980) that fossil fields have mixed configurations in order to be stable in such regions. This statement was verified by numerical simulations and theoretical works (Braithwaite \& Nordlund 2006; Duez et al. 2010), which found that, in non-rotating experiments, the lowest-energy equilibrium states are twisted, non forcefree dipolar fields. Moreover, fossil magnetic fields result from the adjustment of the initial field that has been generated in a previous convective phase (for example during the Pre-Main-Sequence) during the transition from the turbulent convective state to a decaying turbulent one when the stable radiation zone is forming (see Fig. 1; Duez \& Mathis (2010)). The first numerical simulation of this mechanism during the recession of an external convective envelope has been computed by Arlt (2013)). This process is known as turbulent relaxation (e.g. Biskamp 1997). 


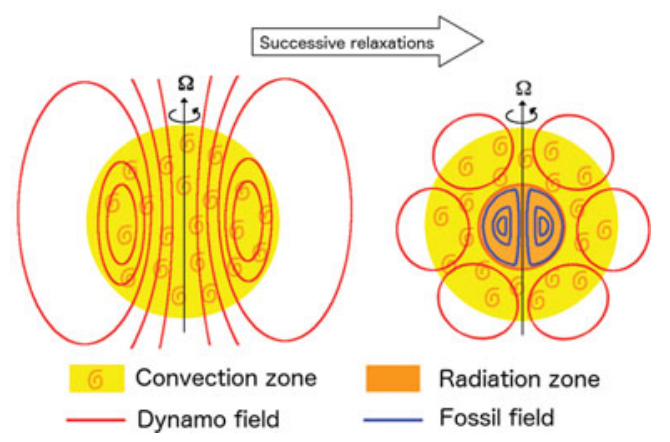

Figure 1. Scheme of the relaxation of fossil magnetic fields during the formation of an internal stably-stratified radiative zone in an initially purely convective star.

\section{Modification of relaxed fossil magnetic fields by rotation}

In this work, we study the impact of rotation on the properties of fossil magnetic fields by considering rotating magnetohydrodynamic, relaxed equilibrium states using the method introduced by Duez \& Mathis (2010). We find that:

- the magnetic field properties are fully determined by the boundary conditions and the properties of their (convective) progenitor, i.e., its magnetic- and cross-helicities and its angular momentum that are almost conserved during the relaxation, while magnetic and kinetic energies decay faster following a selective decay (Biskamp 1997).

- the lowest-energy state for given initial and boundary conditions is the dipolar mode, as in the non-rotating case. In the axisymmetric case, the surface horizontal geometry of the field is dipolar independently of the rotation rate, which modifies only the radial distribution of the magnetic flux. This result is particularly interesting since it matches the observed non-correlation between field geometry and rotation in early-type stars (Wade et al. 2011). It also points the strong difference between magnetic fields resulting from turbulent relaxation and of those generated by $\alpha-\Omega$ dynamos, which have a geometry that is directly modified by rotation (e.g. Petit et al. 2008).

- the field is non force-free (Reisenegger 2009; Braithwaite \& Nordlund 2006; Duez \& Mathis 2010). However, contrary to the non-rotating case, the configuration is nontorque free because the azimutal component of the Lorentz force must counterbalance advection in the rotating case.

\section{References}

Arlt, R. 2013, ArXiv:1309.7126

Biskamp, D. 1997, Nonlinear Magnetohydrodynamics, Cambridge University Press

Braithwaite, J. \& Nordlund, A. 2006, A\&A 450, 1077

Donati, J.-F. \& Landstreet, J. D. 2009, ARA\&A 47, 333

Duez, V., Braithwaite, J., \& Mathis, S. 2010, ApJ (Letters) 724, L34

Duez, V. \& Mathis, S. 2010, A\&A 517, A58

Markey, P. \& Tayler, R. J. 1973, MNRAS 163, 77

Mestel, L. 1999, Stellar magnetism, Oxford University Press

Petit, P., Dintrans, B., Solanki, S. K., et al. 2008, MNRAS 388, 80

Reisenegger, A. 2009, A\&\&A 499, 557

Tayler, R. J. 1973, MNRAS 161, 365

Tayler, R. J. 1980, MNRAS 191, 151

Wade, G. A., Alecian, E., Bohlender, D. A., et al. 2011, in C. Neiner, G. Wade, G. Meynet, \& G. Peters (eds.), IAU Symposium, Vol. 272 of IAU Symposium, pp 118-123 